\title{
The ACE2/Apelin Signaling, MicroRNAs, and Hypertension
}

\author{
Lai-Jiang Chen, ${ }^{1,2,3}$ Ran Xu, ${ }^{1,2}$ Hui-Min Yu, ${ }^{4}$ Qing Chang, ${ }^{1,2}$ and Jiu-Chang Zhong ${ }^{1,2,3}$ \\ ${ }^{1}$ State Key Laboratory of Medical Genomics, Ruijin Hospital, School of Medicine, Shanghai Jiao Tong University, \\ Shanghai 200025, China \\ ${ }^{2}$ Shanghai Key Laboratory of Hypertension, Shanghai Institute of Hypertension, Shanghai 200025, China \\ ${ }^{3}$ Institute of Health Sciences, Shanghai Institute for Biological Sciences, Chinese Academy of Sciences, Shanghai 200025, China \\ ${ }^{4}$ Department of Cardiology, Guangdong General Hospital, Guangdong Academy of Medical Sciences, Guangzhou 510080, China \\ Correspondence should be addressed to Jiu-Chang Zhong; jiuchangzhong@aliyun.com
}

Received 17 December 2014; Accepted 23 February 2015

Academic Editor: Markus Schlaich

Copyright (C) 2015 Lai-Jiang Chen et al. This is an open access article distributed under the Creative Commons Attribution License, which permits unrestricted use, distribution, and reproduction in any medium, provided the original work is properly cited.

\begin{abstract}
The renin-angiotensin aldosterone system (RAAS) plays a pivotal role in the development of hypertension. Angiotensin converting enzyme 2 (ACE2), which primarily metabolises angiotensin (Ang) II to generate the beneficial heptapeptide Ang-(1-7), serves as a negative regulator of the RAAS. Apelin is a second catalytic substrate for ACE2 and functions as an inotropic and cardiovascular protective peptide. The physiological effects of Apelin are exerted through binding to its receptor APJ, a seven-transmembrane G protein-coupled receptor that shares significant homology with the Ang II type 1 receptor (AT1R). The deregulation of microRNAs, a class of short and small noncoding RNAs, has been shown to involve cardiovascular remodeling and pathogenesis of hypertension via the activation of the Ang II/AT1R pathway. MicroRNAs are linked with modulation of the ACE2/Apelin signaling, which exhibits beneficial effects in the cardiovascular system and hypertension. The ACE2-coupled crosstalk among the RAAS, the Apelin system, and microRNAs provides an important mechanistic insight into hypertension. This paper focuses on what is known about the ACE2/Apelin signaling and its biological roles, paying particular attention to interactions and crosstalk among the ACE2/Apelin signaling, microRNAs, and hypertension, aiming to facilitate the exploitation of new therapeutic medicine to control hypertension.
\end{abstract}

\section{Introduction}

Hypertension is a complex cardiovascular disease and contributes to worldwide morbidity and mortality, while its pathogenesis is closely related to abnormalities of the reninangiotensin aldosterone system (RAAS) [1]. Angiotensin converting enzyme 2 (ACE2) serves as a negative regulator of RAAS and cleaves angiotensin (Ang) I to generate the inactive Ang-(1-9) peptide, which can then be converted to Ang-(1-7) by ACE $[2,3]$. Meanwhile, ACE2 can directly metabolize Ang II to generate the beneficial heptapeptide Ang-(1-7), whose actions are often opposite to those attributed to the Ang II and its type 1 receptor (AT1R) signaling. Apelin, a second catalytic substrate for ACE2, has powerful positive inotropic actions and vasodilatation in an endothelium- and nitric oxide(NO-) dependent way [4]. Intriguingly, recent studies have demonstrated that there is a link between the ACE2/Apelin signaling and microRNAs (miRNAs) in the pathogenesis of hypertension. The deregulation of miRNAs expression plays a pivotal role in the development of hypertension. The miRNAs are involved in the regulation of diverse biological processes determining cell proliferation, differentiation, migration, and apoptosis [5-7]. In this review, we focus on the relationship among the ACE2/Apelin signaling, miRNAs, and hypertension and aim to facilitate the exploitation of new therapeutic medicine to control hypertension.

\section{2. miRNAs and Hypertension}

The miRNAs are small double-stranded, noncoding RNAs that function as guide molecules in RNA silencing [5]. miRNAs may regulate gene expression by binding to the $3^{\prime}$ untranslated region ( $3^{\prime}$-UTR) of target messenger RNA transcripts, leading to suppression of protein synthesis or mRNA degradation [5-7]. Previous studies have demonstrated that the majority of miRNAs generate from introns of proteincoding transcripts or noncoding genes in canonical pathways [8]. In general, miRNAs are transcribed by RNA polymerase 
TABLE 1: The RAAS, Apelin/APJ signaling, and microRNAs in hypertension.

\begin{tabular}{|c|c|c|c|}
\hline MicroRNAs & Targets & miRNA function & References \\
\hline miR-143/145 & Klf4; Klf5 & Regulation of VSMCs proliferation & {$[7,16,17]$} \\
\hline miR-365 & Cyclin D1 & Inhibition of VSMCs proliferation & {$[18]$} \\
\hline $\operatorname{miR}-221 / 222$ & p27(Kip1); p57(Kip2) & $\begin{array}{c}\text { Regulation of VSMCs } \\
\text { differentiation and proliferation }\end{array}$ & {$[6,7]$} \\
\hline $\operatorname{miR}-126$ & VEGF; ICAM-1 & Mediator of vascular dysfunction & {$[7,18]$} \\
\hline miR-221/222 & Ets-1; VCAM-1; MCP-1 & $\begin{array}{l}\text { Regulation of inflammation and } \\
\text { vascular remodeling }\end{array}$ & {$[7,18]$} \\
\hline miR-130a & GAX & Regulation of VSMCs proliferation & {$[11]$} \\
\hline miR-145; -27a/27b; & ACE & Inhibition of ACE expression & [20-23] \\
\hline $\operatorname{miR}-421 ;-143$ & ACE2 & Inhibition of ACE2 expression & {$[23,32,33]$} \\
\hline miR-19b; -29; -132/212; -181b; & Ang II & $\begin{array}{l}\text { Promotion of fibrosis and } \\
\text { cardiovascular remodeling }\end{array}$ & {$[24-27,29]$} \\
\hline miR-146a & Ang-(1-7) & Inhibition of inflammation & {$[34]$} \\
\hline miR-155 & AT1R & Inhibition of VSMCs proliferation & {$[19,30,31]$} \\
\hline miR-133a; -208; -1 & Apelin/APJ & $\begin{array}{l}\text { Regulation of cardiovascular } \\
\text { hypertrophy and dysfunction }\end{array}$ & {$[47]$} \\
\hline miR-424; -503 & FGF2; FGFR1 & Inhibition of VSMCs proliferation & {$[48]$} \\
\hline
\end{tabular}

RAAS: renin-angiotensin aldosterone system; Klf4: Krüppel-like factor4; Klf5: Krüppel-like factor5; p27(Kip1): cyclin-dependent kinase inhibitor 1B; p57(Kip2): cyclin-dependent kinase inhibitor 1B; VEGF: vascular endothelial growth factor; Ets-1: E26 transformation-specific sequence 1; VCAM-1: vascular cell adhesion molecule 1; MCP-1: monocyte chemoattractant protein 1; GAX: growth arrest-specific homeobox; ACE: angiotensin converting enzyme; ACE2: angiotensin converting enzyme 2; AT1R: Ang II type 1 receptor; FGF2: fibroblast growth factor 2; FGFR1: fibroblast growth factor receptor 1; VSMCs: vascular smooth muscle cells.

II as a pri-miRNA, which subsequently cleaved into mature miRNA by two RNase enzymes, Drosha and Dicer, in the nucleus and cytoplasm. miRNAs biogenesis is under stringently control and their deregulation links to various diseases [8]. The regulation of miRNAs occurs at multiple levels of their biogenesis. In the process of miRNAs transcription, miRNAs are controlled by RNA polymerase II-associated transcription factors and epigenetic regulators, such as DNA methylation and histone modifications, which are positively or negatively correlated with miRNAs expression, while in the posttranscriptional levels, the modification of miRNAs processing, including RNA editing, methylation, uridylation, adenylation, and RNA decay, contributes to miRNAs regulation. Several critical cell signaling systems have been linked with regulating miRNAs biogenesis at posttranscription levels, such as transforming growth factor- $\beta$ /bone morphogenetic protein pathways and mitogen-activated protein kinases signaling pathways, indicating the diverse possibilities for the regulation of miRNAs biogenesis $[8,9]$.

miRNAs are abundant in all human cells, acting as critical regulators for major cellular functions and miRNAs deregulation associated with a variety of diseases $[5,6]$. Recent studies have demonstrated that miRNAs are associated with the pathogenesis of hypertension [7]. In spontaneously hypertensive rats (SHR) model, the expressions of miR-155 and miR208 were downregulated and negative correlated with blood pressure $[10,11]$. Compared with healthy controls, the plasma levels of miR-126, miR-133, miR-143, and miR-145 were decreased in patients with essential hypertension, combined with enhanced expression of miR-1, miR-296-5p, and miRlet-7e [12-14]. Furthermore, expressions of miR-21, miR-143, and miR-145 were negatively correlated with blood pressure levels, while expressions of miR-9, miR-126, and miR-133 were positively correlated with blood pressure levels [5, 12-14].

It is well established that vascular smooth muscle cells (VSMCs) proliferation, differentiated phenotype, and migration are pivotal cellular events for the development of hypertension [15]. miRNAs are highly expressed in vasculature and modulate diverse vascular cell functions (Table 1) $[5,6]$. Some specific miRNAs have been identified to be involved in VSMCs biology. MiR-143 and MiR-145 are highly expressed in VSMCs and promote VSMCs differentiation and phenotype transformation via the activation of Krüppel-like factor4 (Klf4) and Klf5 (Table 1) [7, 16, 17]. MiR-143 and miR-145 are important stimuli to maintain vascular smooth muscle contractile differentiation, while knockout of them would decrease the blood pressure levels [16]. In addition, miR-365 exerts an antiproliferative role in VSMCs proliferation via the cell cycle regulatory protein cyclin D1 (Table 1) [18]. p27(Kip1) and p57(Kip2) were 2 target genes that were involved in miR-221- and miR-222-mediated effect on VSMC growth and differentiation (Table 1) [6, 7]. These observations indicate that various miRNAs in vasculature may be linked with hypertension and provide novel pharmacologic implications for the prevention and treatment of hypertension.

\section{The ACE/Ang II/AT1R Signaling and MicroRNAs in Hypertension}

Activation of the ACE/Ang II/AT1R signaling promotes inflammation, oxidative stress, and fibrosis linked to hypertension, while inhibition of the ACE/Ang II/AT1R signaling 


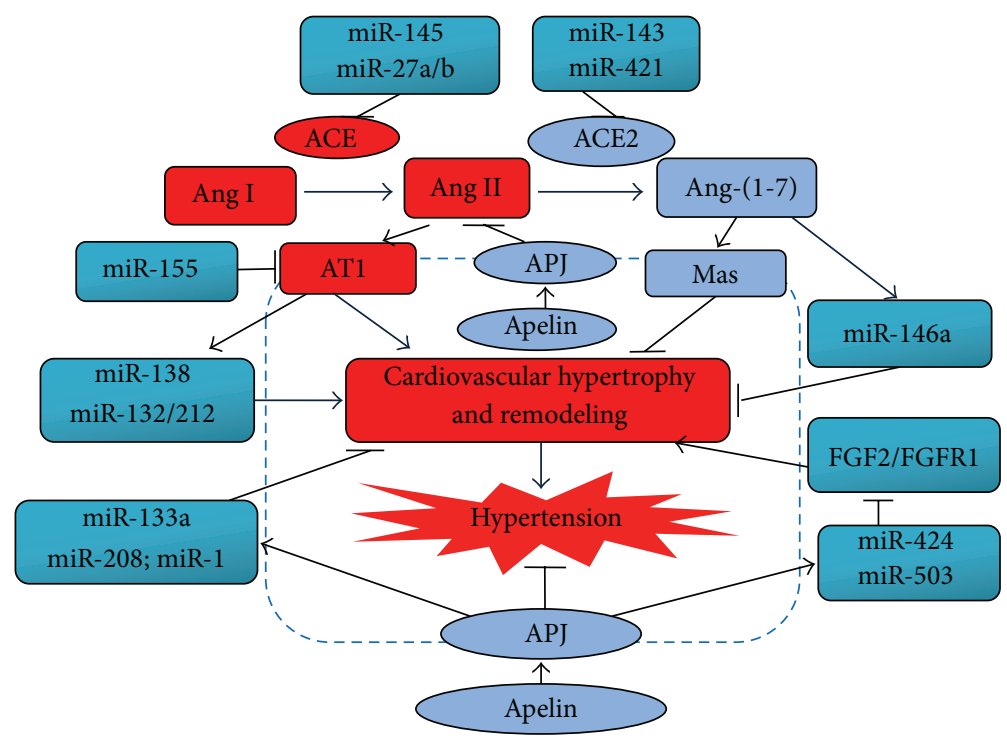

FIgURE 1: The crosstalk between ACE2/Apelin signaling and miRNAs in hypertension. On one hand, the miR-145 and miR-27a/b suppress the action of ACE/Ang II/AT1R, whereas miR-143 and miR-421 serve as negative regulators of ACE2 to modulate the balance between Ang II degradation and Ang-(1-7) generation. Overexpression of miR-155 inhibits the effects mediated by Ang II/AT1R signaling while Ang II regulates miR-138 and miR-132/212 expression via its receptor AT1R, contributing to cardiovascular hypertrophy and remodeling and elevated blood pressure. In contrast, Ang-(1-7) promotes the level of miR-146a, which blocks inflammation and cardiovascular remodeling. On the other hand, the Apelin/APJ signaling regulates the expression of miR-133a, miR-208, and miR-1, functioning as negative regulators for cardiac hypertrophy and contractile function. Moreover, the Apelin/APJ ameliorates cardiovascular hypertrophy and remodeling by modulating miR-424/-503-FGF signaling. ACE: angiotensin converting enzyme; ACE2: angiotensin converting enzyme 2; Ang II: angiotensin; AT1R: angiotensin II type 1 receptor; miRNAs: microRNAs; FGF2: fibroblast growth factor 2; FGFR1: fibroblast growth factor receptor 1.

has been regarded as important pharmacological tools to prevent and treat vascular diseases such as hypertension [1, $3,19]$. Recently, most researches have been focused on the interactions between the ACE/Ang II/AT1R axis and miRNAs in hypertension. The miR-145, miR-27a/27b, and miR-483$3 p$ have been shown to inhibit ACE expression by directly targeting the putative binding sites in the $3^{\prime}$-UTR of ACE transcripts [20-23]. There exists a negative feedback loop between miR-145 and ACE expression, mediating the switch of VSMC phenotypic differentiation to dedifferentiation. The ACE/Ang II signaling mediates various miRNAs expression via the activation of AT1R and these miRNAs, thereby contributing to VSMCs proliferation, cardiovascular remodeling, and hypertension (Figure 1) [24, 25]. In hypertensive status, Ang II-induced miR-130a mediated VSMCs proliferation and vascular remodeling by preventing the expression of growth arrest-specific homeobox (GAX) [11]. In human microvascular endothelial cells, exposure to Ang II increased the stability of HIF1- $\alpha$ with consequent induction of miR-138, which attenuated the bioavailability of $\mathrm{NO}$ and mediated the proinflammatory signal transduction [26]. Eskildsen et al. [27] reported that chronic Ang II infusion enhanced the levels of miR-132/-212 in hypertensive rats, which were prevented by treatment with AT1R blocker. Moreover, they observed that miR-132/-212 fine-tune multiple targets in Ang II signaling led to cardiac hypertrophy and fibrosis [28]. Ang II-mediated miRNAs have been identified to involve hypertension, including miR-19b, miR-29, miR-129-3p, miR-181b, and miR483-3p (Table 1) [22-25, 29]. Kemp et al. [23] investigated that the miR-483-3p could target some components of RAAS directly. In their study, miR-483-3p regulated homeostatic levels of ACE, ACE2, and AT2R, indicating that this miRNA might function as an important regulator of manipulating RAAS. The intrinsic regulation of Ang II-responsive miRNAs on RAS components suggests that the modulation between Ang II and miRNAs is complicated (Table 1). In another work [30], miR-155 was found as a potent regulator of Ang IIinduced VSMCs proliferation by inhibiting AT1R (Figure 1). In hypertensive patients, AT1R expression was negatively correlated with miR-155 expression and the interplay between miR-155 and AT1R was associated with the polymorphism (+1166A/C single-nucleotide polymorphism) located in the $3^{\prime}$-UTR of the AT1R [31]. Although the specific downstream targets of AT1R remain unclear, these findings suggest that miR-155 involve the Ang II/AT1R signaling and relate to vascular diseases.

\section{The ACE2/Ang-(1-7)/Mas Signaling and MicroRNAs in Hypertension}

The classical pathway of the RAS involving the ACE-Ang II-AT1 receptor axis is now antagonized by the second arm constituted by the ACE2-Ang-(1-7)-Mas receptor axis, exerting protective effects via modulating the inflammation, fibrosis, and vascular remodeling in hypertension [1-3]. Recent studies have shown the link between the ACE2/Ang(1-7)/Mas signaling and miRNAs in hypertension (Table 1 and Figure 1) [32-34]. miRNA-targeting prediction algorithms 
revealed pupative binding sites for a variety of miRNAs in the $3^{\prime}$-UTR of ACE2 transcript, while only several miRNAs have been identified as regulators in inhibiting ACE2 expression $[23,32,33]$. In SHR model [33], exercise training significantly decreased the blood pressure levels and miR-143 expression levels in aortas in association with a drastic augment in circulating ACE2 and Ang-(1-7) levels, indicating the regulatory roles of miR-143 in the ACE2/Ang-(1-7) signaling (Table 1 and Figure 1). By establishing luciferase report system containing ACE2 $3^{\prime}$-UTR, both miR-421 and miR-483-3p strikingly decreased ACE2 protein levels while loss of the two miRNAs reversed these effects, implying that miR-421 and miR-483-3p modulated ACE2 expression via translational repression rather than degraded the transcripts (Table 1) $[23,32]$. In human aortic endothelial cells (Table 1), Ang-(1-7) decreased glycated albumin-mediated vascular inflammation and improved the vascular function through modulating the expression of miR-146a [34]. In addition, treatment with telmisartan modulated the level of miR-146a/b, along with the improvement of the ACE2/Ang-(1-7) levels and attenuation of vascular remodeling in hypertension [35-38]. Satoh et al. [39] reported that blockade of RAAS in coronary artery disease patients decreased protein levels of TLR4 and upregulated TLR4-responsive miRNAs expression including miR-31, miR-181a, miR-16, and miR-145 compared to controls. Although the related mechanism has not been identified, these researches permit speculation that RAAS blockades may exert cardiovascular protective effects by modulating the ACE2/Ang-(1-7) signaling and miRNAs levels. Taken together, there are potential interactions between the ACE2/Ang-(1-7) signaling and miRNAs in hypertension.

\section{The Apelin/APJ Signaling and MicroRNAs in Hypertension}

Apelin is a second catalytic substrate for ACE2 and functions as an inotropic and cardioprotective peptide [4, 38, 40-43]. The physiological effects of Apelin are exerted through binding to its receptor APJ, a seven-transmembrane G proteincoupled receptor that shares significant homology with the AT1R. Intriguingly, there is $54 \%$ sequence homology of the transmembrane regions between the APJ and AT1R and the anatomical distribution of both receptors and peptides overlaps in the cardiovascular system [42]. However, the effects of Apelin is independent of AT1 receptor and Ang II cannot bind to the APJ, although both of Ang II and Apelin can be degraded by ACE2 [38, 43]. The Apelin/APJ system has been shown to be involved in a wide range of pathophysiology effects in cardiovascular system, which is a necessary process in the initiation and development of various cardiovascular diseases such as pulmonary hypertension and essential hypertension [40, 41, 43]. Apelin levels were reduced in patients with essential hypertension independent of left ventricular systolic and diastolic dysfunction [44]. Treatment with Apelin has been shown to lower systolic blood pressure levels in hypertensive rats via a NO-dependent signaling $[45,46]$. miRNAs are involved in VSMCs proliferation, cardiovascular remodeling, and the pathogenesis of hypertension (Table 1) [7, 16-18]. Ceylan-Isik et al. [47] found that treatment with Apelin strikingly prevented high fat dietinduced cardiac hypertrophy and contractile dysfunction associated with increased levels of miR-1, miR-208, and miR133a. In addition, Apelin treatment significantly reduced the expression of fibroblast growth factor 2 (FGF2) and FGF receptor 1 (FGFR1) by regulating miR-424 and miR-503, contributing to the attenuation of VSMCs proliferation (Table 1 and Figure 1). Conversely, Apelin deficiency resulted in a marked increase in FGF2 expression and a reduction in expression of miR-424 and miR-503 that worsen pulmonary arterial hypertension in experimental models, suggesting the regulatory roles of Apelin in maintaining vascular homeostasis via the miR-424/503-FGF signaling [48]. Intriguingly, Apelin upregulated ACE2 in the failing hearts, coupled with upregulation of miR-424 and miR-503, whereas Ang-(17) administration improved cardiovascular dysfunction of Apelin-deficient mice in vivo [40, 48]. Moreover, the ApelinAPJ increased ACE2 activity and improved cardiovascular remodeling independently of AT1R signaling [40]. Both ACE2 and Apelin exhibit beneficial effects in the cardiovascular system, and recombinant ACE2 has recently been shown as a candidate therapeutic for treating hypertension in animal models [38, 40, 41]. The crosstalk between the ACE2/Apelin signaling and miRNAs provides an important mechanistic insight into hypertension and may lead to the development of new therapeutic regimens.

\section{Conclusions, Limitations, and Perspectives}

The discovery of the interaction between the ACE2/Apelin signaling and miRNAs during hypertension is an exciting affair in hypertensive research. ACE2 is a pleiotropic monocarboxypeptidase responsible for the conversion of Ang II to Ang-(1-7), thereby functioning as a negative regulator of the RAAS in cardiovascular system $[3,19,38]$. Apelin, as the second substrate of ACE2, exerts a cardiovascular protective effect including lowering blood pressure and improving cardiac contractility [4, 38-41]. The ACE2/Apelin signaling exhibits beneficial effects in the cardiovascular system and hypertension $[3,4,38,40]$. The ACE2-coupled crosstalk among the RAAS, the Apelin system, and miRNAs provides an important mechanistic insight into hypertension.

Although most researches indicate the interactions among RAAS, ACE2/Apelin, and miRNAs in hypertension, these correlations are just in statistic level. These researches just found the linkage between the ACE2/Apelin and miRNAs in patients with hypertension or animal models of hypertension or in vitro VSMCs. However, the ACE2/Apelinrelated miRNAs are really big families which contain "good" or "bad" components and may have additional control mechanisms beyond our current understanding. Thus, the corresponding targets and functional roles of the ACE2/Apelinrelated miRNAs still need to be identified. The investigation of the relationship between the ACE2/Apelin signaling and miRNAs is just a beginning; there is still a long way to go. Further studies will be needed to explore the interactions and precise mechanisms of the ACE2/Apelin signaling and evaluate the role of validated and predicted targets of miRNAs 
in hypertension. Targeting the ACE2/Apelin signaling and miRNAs could lead to the development of a novel therapeutic approach for patients with hypertension and other vascular diseases related to cardiovascular remodeling.

\section{Conflict of Interests}

On behalf of all authors, the corresponding author states that there is no conflict of interests.

\section{Authors' Contribution}

Lai-Jiang Chen and Ran Xu contributed equally to this work.

\section{Acknowledgments}

This work was supported by the National Basic Research Program of China (973 Program; 2014CB542300), the Training Program of the National Major Research Plan (91339108), and General Program (81370362, 81273599, and 81170246) of the National Natural Science Foundation of China and Scientific Research Project of Health Bureau of Shanghai (201440368).

\section{References}

[1] J. Y. Moon, "Recent update of renin-angiotensin-aldosterone system in the pathogenesis of hypertension," Electrolytes \& Blood Pressure, vol. 11, no. 2, pp. 41-45, 2013.

[2] V. B. Patel, J.-C. Zhong, D. Fan et al., "Angiotensin-converting enzyme 2 is a critical determinant of angiotensin II-induced loss of vascular smooth muscle cells and adverse vascular remodeling," Hypertension, vol. 64, no. 1, pp. 157-164, 2014.

[3] Z. Zhang, L. Chen, J. Zhong, P. Gao, and G. Y. Oudit, "ACE2/ Ang-(1-7) signaling and vascular remodeling," Science China Life Sciences, vol. 57, no. 8, pp. 802-808, 2014.

[4] X.-H. Yu, Z.-B. Tang, L.-J. Liu et al., "Apelin and its receptor APJ in cardiovascular diseases," Clinica Chimica Acta, vol. 428, pp. $1-8,2014$.

[5] X. Song, D. Shan, J. Chen, and Q. Jing, "miRNAs and lncRNAs in vascular injury and remodeling," Science China Life Sciences, vol. 57, no. 8, pp. 826-835, 2014.

[6] E. Bronze-da-Rocha, "MicroRNAs expression profiles in cardiovascular diseases," BioMed Research International, vol. 2014, Article ID 985408, 23 pages, 2014.

[7] S. Bátkai and T. Thum, "MicroRNAs in hypertension: mechanisms and therapeutic targets," Current Hypertension Reports, vol. 14, no. 1, pp. 79-87, 2012.

[8] M. Ha and V. N. Kim, "Regulation of microRNA biogenesis," Nature Reviews Molecular Cell Biology, vol. 15, no. 8, pp. 509$524,2014$.

[9] A. Saj and E. C. Lai, "Control of microRNA biogenesis and transcription by cell signaling pathways," Current Opinion in Genetics and Development, vol. 21, no. 4, pp. 504-510, 2011.

[10] C. C. Xu, W. Q. Han, B. Xiao, N. N. Li, D. L. Zhu, and P. J. Gao, "Differential expression of microRNAs in the aorta of spontaneously hypertensive rats," Sheng Li Xue Bao, vol. 60, no. 4, pp. 553-560, 2008.
[11] W.-H. Wu, C.-P. Hu, X.-P. Chen et al., "MicroRNA-130a mediates proliferation of vascular smooth muscle cells in hypertension," American Journal of Hypertension, vol. 24, no. 10, pp.10871093, 2011.

[12] J. E. Kontaraki, M. E. Marketou, E. A. Zacharis, F. I. Parthenakis, and P. E. Vardas, "Differential expression of vascular smooth muscle-modulating microRNAs in human peripheral blood mononuclear cells: novel targets in essential hypertension," Journal of Human Hypertension, vol. 28, no. 8, pp. 510-516, 2014.

[13] J. E. Kontaraki, M. E. Marketou, E. A. Zacharis, F. I. Parthenakis, and P. E. Vardas, "MicroRNA-9 and microRNA-126 expression levels in patients with essential hypertension: potential markers of target-organ damage," Journal of the American Society of Hypertension, vol. 8, no. 6, pp. 368-375, 2014.

[14] S. Li, J. Zhu, W. Zhang et al., "Signature microRNA expression profile of essential hypertension and its novel link to human cytomegalovirus infection," Circulation, vol. 124, no. 2, pp. 175184, 2011.

[15] E. Schulz, T. Gori, and T. Münzel, "Oxidative stress and endothelial dysfunction in hypertension," Hypertension Research, vol. 34, no. 6, pp. 665-673, 2011.

[16] L. Elia, M. Quintavalle, J. Zhang et al., "The knockout of miR-143 and -145 alters smooth muscle cell maintenance and vascular homeostasis in mice: correlates with human disease," Cell Death and Differentiation, vol. 16, no. 12, pp. 1590-1598, 2009.

[17] T. Boettger, N. Beetz, S. Kostin et al., "Acquisition of the contractile phenotype by murine arterial smooth muscle cells depends on the Mir143/145 gene cluster," The Journal of Clinical Investigation, vol. 119, no. 9, pp. 2634-2647, 2009.

[18] M.-H. Kim, O. Ham, S.-Y. Lee et al., "MicroRNA-365 inhibits the proliferation of vascular smooth muscle cells by targeting cyclin D1," Journal of Cellular Biochemistry, vol. 115, no. 10, pp. 1752-1761, 2014.

[19] B. Song, Z.-Z. Zhang, J.-C. Zhong et al., "Loss of angiotensinconverting enzyme 2 exacerbates myocardial injury via activation of the CTGF-fractalkine signaling pathway," Circulation Journal, vol. 77, no. 12, pp. 2997-3006, 2013.

[20] B. Hu, J. T. Song, H. Y. Qu et al., "Mechanical stretch suppresses microRNA-145 expression by activating extracellular signalregulated kinase $1 / 2$ and upregulating angiotensin- converting enzyme to alter vascular smooth muscle cell phenotype," PLoS ONE, vol. 9, no. 5, Article ID e96338, 2014.

[21] K. Kohlstedt, C. Trouvain, T. Boettger, L. Shi, B. Fisslthaler, and I. Fleming, "AMP-activated protein kinase regulates endothelial cell angiotensin-converting enzyme expression via p53 and the post-transcriptional regulation of microRNA-143/145," Circulation Research, vol. 112, no. 8, pp. 1150-1158, 2013.

[22] R. Goyal, D. Goyal, A. Leitzke, C. P. Gheorghe, and L. D. Longo, "Brain renin-angiotensin system: fetal epigenetic programming by maternal protein restriction during pregnancy," Reproductive Sciences, vol. 17, no. 3, pp. 227-238, 2010.

[23] J. R. Kemp, H. Unal, R. Desnoyer, H. Yue, A. Bhatnagar, and S. S. Karnik, "Angiotensin II-regulated microRNA 483-3p directly targets multiple components of the rennin-angiotensin system," Journal of Molecular and Cellular Cardiology, vol. 75, pp. 25-39, 2014.

[24] X. Jiang, Q. Ning, and J. Wang, "Angiotensin II induced differentially expressed microRNAs in adult rat cardiac fibroblasts," Journal of Physiological Sciences, vol. 63, no. 1, pp. 31-38, 2013.

[25] P. L. Jeppesen, G. L. Christensen, M. Schneider et al., "Angiotensin II type 1 receptor signalling regulates microRNA 
differentially in cardiac fibroblasts and myocytes," British Journal of Pharmacology, vol. 164, no. 2, pp. 394-404, 2011.

[26] A. Sen, P. Most, and K. Peppel, "Induction of microRNA-138 by pro-inflammatory cytokines causes endothelial cell dysfunction," FEBS Letters, vol. 588, no. 6, pp. 906-914, 2014.

[27] T. V. Eskildsen, P. L. Jeppesen, M. Schneider et al., "Angiotensin II regulates microRNA-132/-212 in hypertensive rats and humans," International Journal of Molecular Sciences, vol. 14, no. 6, pp. 11190-11207, 2013.

[28] T. V. Eskildsen, M. Schneider, M. B. Sandberg et al., "The microRNA-132/212 family fine-tunes multiple targets in Angiotensin II signalling in cardiac fibroblasts," Journal of the ReninAngiotensin-Aldosterone System, 2014.

[29] S. Gao, T.-W. Liu, Z. Wang et al., "Downregulation of MicroRNA-19b contributes to angiotensin II-induced overexpression of connective tissue growth factor in cardiomyocytes," Cardiology, vol. 127, no. 2, pp. 114-120, 2014.

[30] L.-X. Yang, G. Liu, G.-F. Zhu et al., "MicroRNA-155 inhibits angiotensin II-induced vascular smooth muscle cell proliferation," Journal of the Renin Angiotensin Aldosterone System, vol. 15, no. 2, pp. 109-116, 2014.

[31] G. Ceolotto, I. Papparella, A. Bortoluzzi et al., "Interplay between miR-155, AT1R A1166C polymorphism, and AT1R expression in young untreated hypertensives," The American Journal of Hypertension, vol. 24, no. 2, pp. 241-246, 2011.

[32] D. W. Lambert, L. A. Lambert, N. E. Clarke, N. M. Hooper, K. E. Porter, and A. J. Turner, "Angiotensin-converting enzyme 2 is subject to post-transcriptional regulation by miR-421," Clinical Science, vol. 127, no. 4, pp. 243-249, 2014.

[33] Q. Gu, B. Wang, X.-F. Zhang, Y.-P. Ma, J.-D. Liu, and X.-Z. Wang, "Contribution of renin-angiotensin system to exerciseinduced attenuation of aortic remodeling and improvement of endothelial function in spontaneously hypertensive rats," Cardiovascular Pathology, vol. 23, no. 5, pp. 298-305, 2014.

[34] H.-J. Wang, W.-Y. Lo, and L.-J. Lin, "Angiotensin-(1-7) decreases glycated albumin-induced endothelial interleukin6 expression via modulation of miR-146a," Biochemical and Biophysical Research Communications, vol. 430, no. 3, pp. 1157$1163,2013$.

[35] Y. Takahashi, M. Satoh, Y. Minami, T. Tabuchi, T. Itoh, and M. Nakamura, "Expression of miR-146a/b is associated with the Toll-like receptor 4 signal in coronary artery disease: effect of renin-angiotensin system blockade and statins on miRNA146a/b and Toll-like receptor 4 levels," Clinical Science, vol. 119, no. 9, pp. 395-405, 2010.

[36] C. M. Ferrario, J. Jessup, M. C. Chappell et al., "Effect of angiotensin-converting enzyme inhibition and angiotensin II receptor blockers on cardiac angiotensin-converting enzyme 2," Circulation, vol. 111, no. 20, pp. 2605-2610, 2005.

[37] J. C. Zhong, J. Y. Ye, H. Y. Jin et al., “Telmisartan attenuates aortic hypertrophy in hypertensive rats by the modulation of ACE2 and profilin-1 expression," Regulatory Peptides, vol. 166, no. 1-3, pp. 90-97, 2011.

[38] J. Zhong, R. Basu, D. Guo et al., "Angiotensin-converting enzyme 2 suppresses pathological hypertrophy, myocardial fibrosis, and cardiac dysfunction," Circulation, vol. 122, no. 7, pp. 717-728, 2010.

[39] M. Satoh, Y. Takahashi, T. Tabuchi et al., "Circulating Toll-like receptor 4-responsive microRNA panel in patients with coronary artery disease: results from prospective and randomized study of treatment with renin-angiotensin system blockades," Clinical Science, vol. 128, no. 8, pp. 483-491, 2015.
[40] T. Sato, T. Suzuki, H. Watanabe et al., "Apelin is a positive regulator of ACE2 in failing hearts," Journal of Clinical Investigation, vol. 123, no. 12, pp. 5203-5211, 2013.

[41] N. E. Clarke and A. J. Turner, "Angiotensin-converting enzyme 2: the first decade," International Journal of Hypertension, vol. 2012, Article ID 307315, 12 pages, 2012.

[42] K. Tatemoto, M. Hosoya, Y. Habata et al., "Isolation and characterization of a novel endogenous peptide ligand for the human APJ receptor," Biochemical and Biophysical Research Communications, vol. 251, no. 2, pp. 471-476, 1998.

[43] J. C. Zhong, X. Y. Yu, Y. Huang, L. M. Yung, C. W. Lau, and S. G. Lin, "Apelin modulates aortic vascular tone via endothelial nitric oxide synthase phosphorylation pathway in diabetic mice," Cardiovascular Research, vol. 74, no. 3, pp. 388395, 2007.

[44] M. Przewlocka-Kosmala, T. Kotwica, A. Mysiak, and W. Kosmala, "Reduced circulating apelin in essential hypertension and its association with cardiac dysfunction," Journal of Hypertension, vol. 29, no. 5, pp. 971-979, 2011.

[45] H. Najafipour, A. Soltani Hekmat, A. A. Nekooian, and S. Esmaeili-Mahani, "Apelin receptor expression in ischemic and non- ischemic kidneys and cardiovascular responses to apelin in chronic two-kidney-one-clip hypertension in rats," Regulatory Peptides, vol. 178, no. 1-3, pp. 43-50, 2012.

[46] R. Akcilar, S. Turgut, V. Caner et al., "Apelin effects on blood pressure and RAS in DOCA-salt-induced hypertensive rats," Clinical and Experimental Hypertension, vol. 35, no. 7, pp. 550557, 2013.

[47] A. F. Ceylan-Isik, M. R. Kandadi, X. Xu et al., "Apelin administration ameliorates high fat diet-induced cardiac hypertrophy and contractile dysfunction," Journal of Molecular and Cellular Cardiology, vol. 63, pp. 4-13, 2013.

[48] J. Kim, Y. Kang, Y. Kojima et al., "An endothelial apelin-FGF link mediated by miR-424 and miR-503 is disrupted in pulmonary arterial hypertension," Nature Medicine, vol. 19, no. 1, pp. 74-82, 2013. 


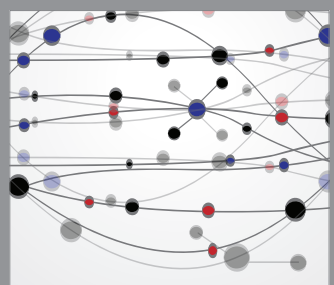

The Scientific World Journal
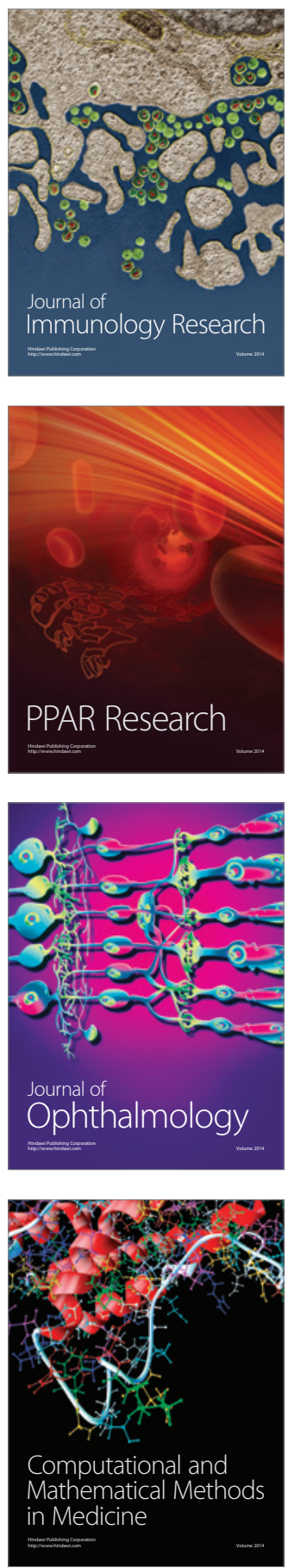

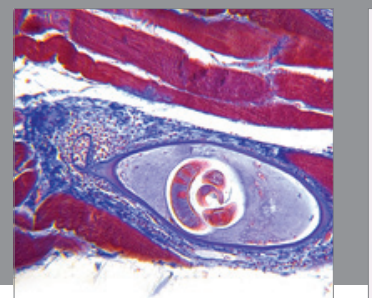

Gastroenterology

Research and Practice
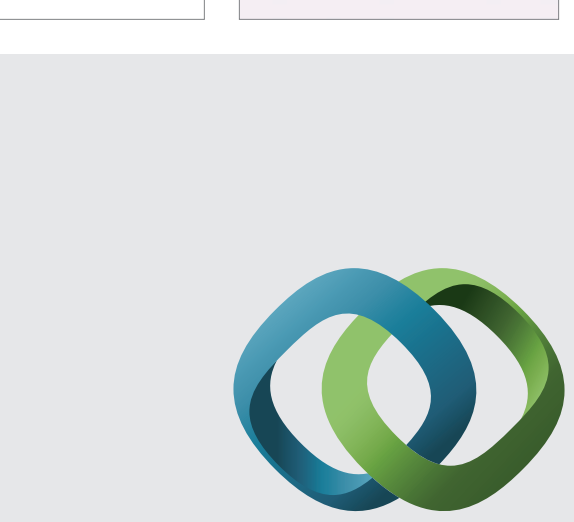

\section{Hindawi}

Submit your manuscripts at

http://www.hindawi.com
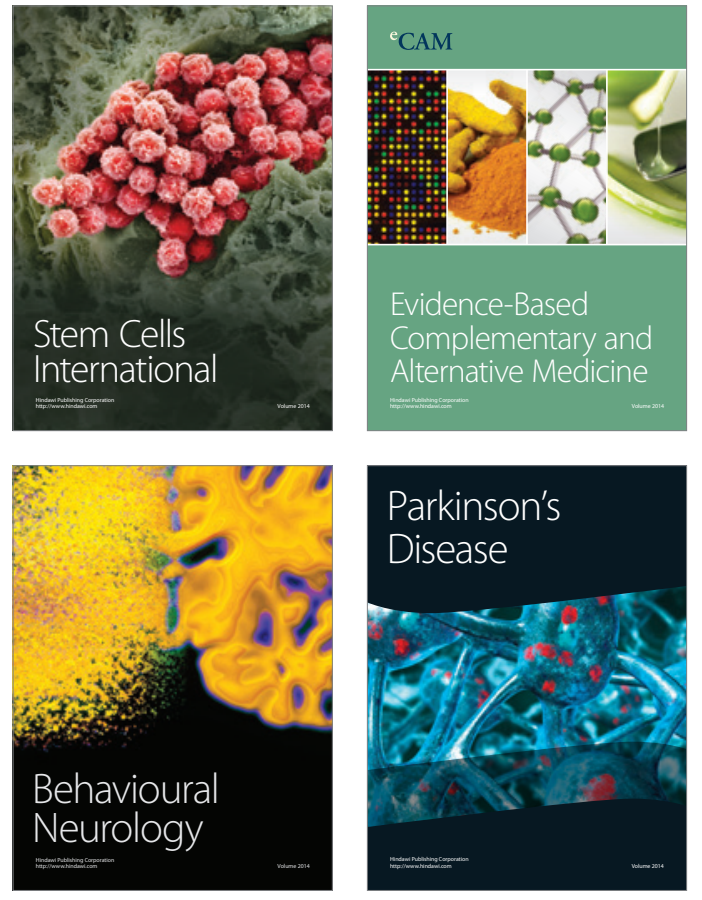
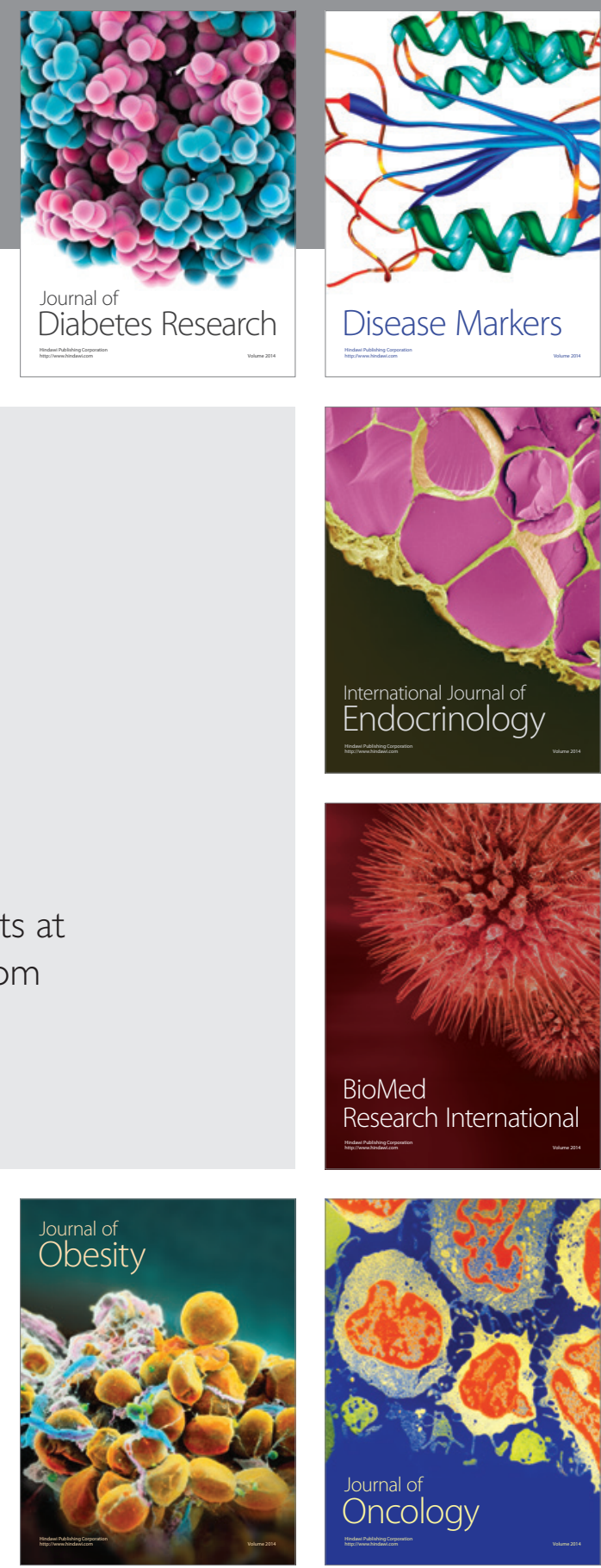

Disease Markers
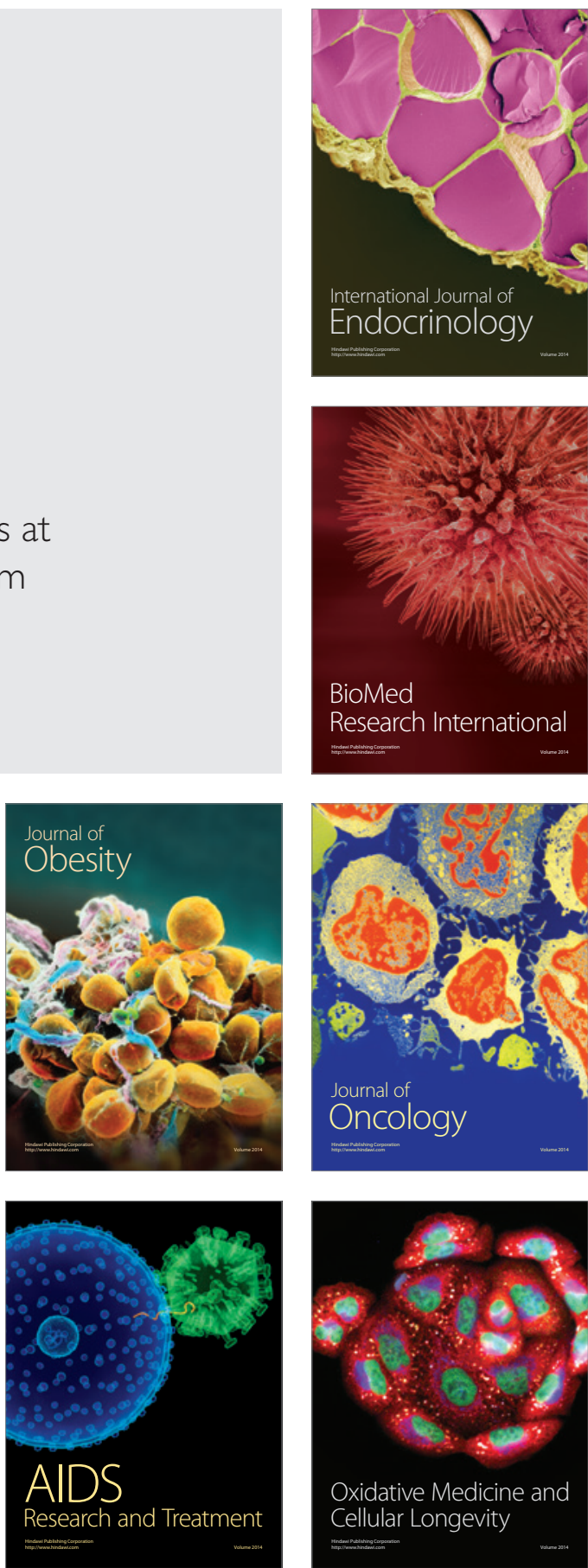